\title{
Second-Generation Trabecular Micro-Bypass Stents as Standalone Treatment for Glaucoma: A 36-Month Prospective Study
}

\author{
Fritz H. Hengerer - Gerd U. Auffarth - Christoffer Riffel • \\ Ina Conrad-Hengerer
}

Received: March 13, 2019 / Published online: May 22, 2019 (C) The Author(s) 2019

\section{ABSTRACT}

Introduction: To evaluate long-term outcomes following stand-alone implantation of two second-generation trabecular micro-bypass stents

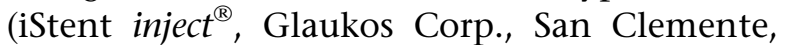
CA, USA) in eyes with predominantly primary open-angle glaucoma (POAG) and considerable preoperative disease burden.

Methods: Eyes with POAG, pseudoexfoliative glaucoma (PXG), appositional narrow-angle glaucoma (NAG, with open-angle configuration in the area of implantation), or secondary glaucoma were included in this prospective, non-randomized, consecutive case series. All eyes underwent $a b$ interno iStent inject implantation as a sole procedure. Assessments through 36 months included IOP, medications, corrected distance visual acuity (CDVA), secondary glaucoma surgeries, and complications and adverse events.

Enhanced Digital Features To view enhanced digital features for this article go to https://doi.org/10.6084/ m9.figshare.8067734.

\footnotetext{
F. H. Hengerer $(\bowtie)$ · G. U. Auffarth · C. Riffel ·

I. Conrad-Hengerer

University Eye Hospital, University of Heidelberg,

Heidelberg, Germany

e-mail: f.hengerer@buergerhospital-ffm.de;

mail@augenarzt-trebur.de

F. H. Hengerer

Department of Ophthalmology, Buergerhospital,

Frankfurt, Germany
}

Results: Two iStent inject stents were implanted in 44 consecutive eyes $(\mathrm{POAG}=38, \mathrm{PXG}=4$, appositional $\mathrm{NAG}=1$, secondary neovascular glaucoma $=1$ ) of 31 patients, and 33 eyes had 36-month follow-up data. Preoperative mean IOP was $25.3 \pm 6.0 \mathrm{mmHg}$ on a mean of $2.98 \pm 0.88$ medications, with $75 \%$ of eyes on 3-5 medications, no eyes medication-free, and $50 \%$ of eyes with history of prior glaucoma surgery. At 36 months postoperatively, mean IOP reduced by $42 \%$ to $14.6 \pm 2.0 \mathrm{mmHg}$ $(p<0.0001)$ and $87.9 \%$ of eyes achieved an IOP reduction of $\geq 20 \%$ versus preoperatively. In addition, $97 \%$ of eyes reached IOP $\leq 18 \mathrm{mmHg}$ (vs. 9.1\% preoperatively; $p<0.0001$ ) and $70.0 \%$ of eyes reached IOP $\leq 15 \mathrm{mmHg}$ (vs. $2.3 \%$ preoperatively; $p<0.0001)$. Mean medication burden decreased by $82 \%$ to $0.55 \pm 0.79$ $(p<0.0001)$, and $61 \%$ of eyes became medication-free. All eyes maintained or decreased their 36-month medication burden versus preoperatively. Safety was favorable, including minimal adverse events and stable CDVA through 36 months postoperatively.

Conclusion: This real-world cohort of glaucomatous eyes with substantial preoperative disease burden experienced significant, sustained, safe IOP and medication reductions through 36 months following stand-alone iStent inject implantation.

Funding: Article processing charges and writing assistance were provided by Glaukos Corp. (San Clemente, CA, USA). 
Keywords: Glaucoma; IStent inject; Intraocular pressure; Microinvasive glaucoma surgery (MIGS); Second generation; Stand alone; Stent; Trabecular; Medication

\section{INTRODUCTION}

Glaucoma consistently ranks as one of the leading causes of all blindness worldwide and is the single biggest cause of irreversible blindness $[1,2]$. Population estimates reveal formidable numbers of patients affected by glaucoma, with rates increasing as life expectancies lengthen. To prevent visual deterioration and optic nerve damage, patients and their doctors initiate treatment, the cornerstone of which is the reduction of intraocular pressure (IOP). Medications typically are the first line of treatment, although their effectiveness is limited by local and systemic side effects, ocular surface damage, cost, complex dosing regimens, and suboptimal patient adherence [3-11]. Laser trabeculoplasty often is completed alongside or after medications, although it induces inflammation and its IOP-lowering impact has a limited time frame [12].

In later stages of the disease, traditional filtering surgeries such as tube shunt implantation or trabeculectomy are the main treatment modalities; however failure rates and long-term complications (e.g., endophthalmitis, hypotony, choroidal detachment, bleb leak, or infection) are a real concern [13-15]. If dramatic and immediate IOP reduction is needed, then these risks may be appropriate. However, for a large portion of the glaucoma population-particularly in eyes that need additional intervention beyond medication and/or lasers, but that do not yet warrant the risks of filtering surgery-a surgical treatment with a more favorable benefit-to-risk ratio may be preferable.

Micro-invasive glaucoma surgery (MIGS) offers such a treatment, as it has shown consistent reductions in IOP and medication burden, while also maintaining favorable longterm safety [16]. A sizable body of evidence has shown that the eyes benefiting from this treatment can include those with more moderate and severe stages of glaucoma as well as mild glaucoma; and they may include eyes with newly diagnosed, treatment-naive glaucoma, in addition to those that already tried (and failed) prior medications and/or laser procedures.

The first MIGS implant was the iStent ${ }^{\circledR}$ Trabecular Micro-Bypass (Glaukos Corp., San Clemente, CA, USA; FDA 2012, CE 2004). To date, this device has amassed a sizable longterm evidence base regarding its safety and performance in eyes with open-angle glaucoma, in settings both with and without cataract surgery. Most studies of the device have assessed outcomes in mild to moderate glaucoma [17-27], but other patient populations increasingly have been the subject of evaluation, such as pseudoexfoliative glaucoma, severe or refractory glaucoma, or newly diagnosed, treatment-naïve glaucoma [28-33]. Another growing and relevant area of investigation has been economics outcomes research [34-39], which consistently has shown favorable cost-effectiveness of the iStent in various healthcare models around the world.

The newest US FDA-approved device in the MIGS treatment area is the second-generation iStent inject ${ }^{\circledR}$ Trabecular Micro-Bypass (Glaukos; FDA 2018, CE 2010), which includes two trabecular stents designed to reduce IOP by bolstering aqueous outflow through the trabecular meshwork into Schlemm's canal (Figs. 1-2). In studies both with and without cataract surgery, and in both investigational and real-world settings, the $a b$ interno-implanted iStent inject has demonstrated clinically significant reductions in IOP and medications over the long term [40-51]. These performance outcomes have been accompanied by favorable safety and a correspondingly advantageous benefit-to-risk profile.

In the present prospective cohort, we evaluate whether stand-alone second-generation trabecular bypass stent implantation is a durable, effective, and safe method to reduce IOP and medications in glaucomatous eyes with a substantial preoperative disease burden. This intervention was completed by a single surgeon within a real-world clinical setting and with a relatively long period of follow-up, providing relevant data to doctors and patients evaluating their glaucoma treatment options. 

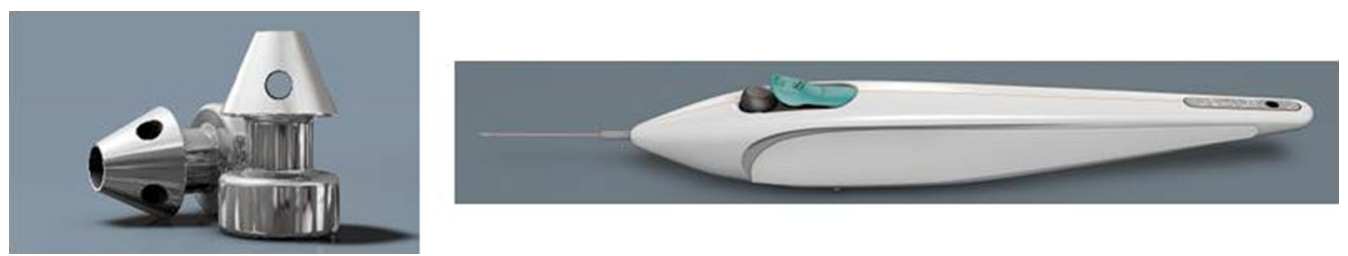

Fig. 1 iStent inject $^{\circledR}$ and iStent inject ${ }^{\circledR}$ Trabecular Micro-Bypass Stent System. Provided by Glaukos Corp.
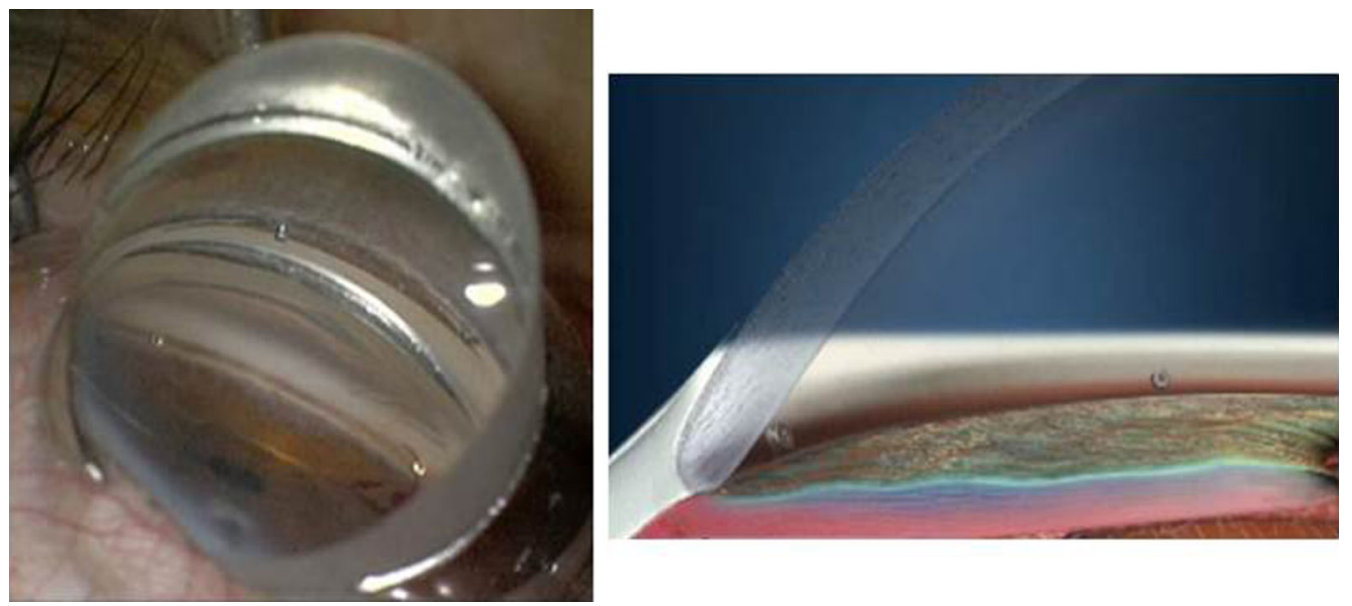

Fig. 2 iStent inject ${ }^{\circledR}$ implantation: placement of two stents in the anterior chamber angle. Provided by Glaukos Corp.

\section{METHODS}

\section{Study Design}

This prospective, non-randomized, consecutive case series included eyes with various types of glaucoma that underwent implantation of two second-generation trabecular micro-bypass stents as a stand-alone procedure. One surgeon (F.H.) completed all surgeries during a 39-month period at an academic ophthalmology center in Heidelberg, Germany. Subjects were required to have glaucoma [including primary open-angle glaucoma (POAG), appositional narrow-angle glaucoma (NAG, defined as angle Shaffer grade 2, with an open angle in the area of stent implantation), pseudoexfoliative glaucoma (PXG), or secondary glaucoma], to be eligible for iStent inject surgery, and to have experienced glaucoma progression despite prior medical and/or surgical glaucoma treatment. Exclusion criteria included active intraocular inflammation, corneal and/or media opacities preventing the gonioscopic view, angle closure in the area of stent implantation, pregnancy, age $<18$ years, or congenital glaucoma.

Effectiveness outcomes consisted of IOP (3 measurements per time point, measured by Goldmann applanation) and topical ocular hypotensive medications. Baseline IOP was determined by IOP readings at two or more preoperative visits. Safety outcomes included CDVA, intraoperative and postoperative adverse events, and secondary surgical interventions. During the follow-up period, testing included visual fields every 6 months, OCT RNFL every 12 months, and optic nerve examination at every visit. Further surgical procedures were considered if visual field, OCT, or optic nerve findings were concerning for glaucoma progression and/or if IOP was inadequately reduced by iStent inject surgery (per surgeon discretion).

All procedures performed were in accordance with the ethical standards of the institutional and/or national research committee [the Institutional Review Board (IRB) of the University of Heidelberg] and with the 1964 Helsinki 
Declaration and its later amendments or comparable ethical standards. Informed consent was obtained from all individual participants included in the study. No clinical trial registration was required for the study. Patients have been followed through 36 months postoperatively, and follow-up is ongoing.

\section{Stent Description, Surgical Technique, and Perioperative Medication}

iStent inject implantation was completed as a sole procedure (i.e., without cataract surgery), using the standard implantation technique described previously [40, 43, 45]. In brief, the procedure consists of the following: after pupillary miosis and anterior chamber filling with a medium viscous ocular surgery viscoelastic device (OVD), the single-use, stainless steel injector is advanced through a temporal corneal incision to the nasal Schlemm's canal, where two pre-loaded titanium stents are implanted $a b$ interno approximately two clockhours apart (Figs. 1-2). Each heparin-coated, $360 \mu \mathrm{m} \times 230-\mu \mathrm{m}$ stent has a symmetric design (compatible with right or left eyes) and several lateral outlet lumens designed to decrease IOP by bolstering aqueous outflow from the anterior chamber to Schlemm's canal. OVD is removed by bimanual irrigation and aspiration. After surgery, patients received 1 week of topical antibiotic (ciprofloxacin) and 4 weeks of topical anti-inflammatory medication (dexamethasone).

\section{Data analyses}

Descriptive statistics were used to summarize mean IOP and number of medications from the preoperative visit through 36 months postoperatively. Preoperative and 36-month proportional analyses were completed for percent of eyes with IOP $\leq 18 \mathrm{mmHg}$, IOP $<15 \mathrm{mmHg}$, and $\geq 20 \%$ IOP reduction; eyes on $0,1,2$, or $\geq 3$ medications; and eyes with reduced medications versus preoperatively. A paired $t$-test was used to compare preoperative versus month 36 mean IOP and number of medications, and the
McNemar test was used to compare preoperative and month 36 proportional IOP outcomes.

\section{RESULTS}

\section{Subject Accountability, Demographics, and Preoperative Parameters}

A total of 44 consecutive eyes of 31 patients were evaluated in this cohort, with glaucoma diagnoses including POAG $(n=38)$, PXG $(n=4)$, appositional NAG $(n=1)$, and secondary (neovascular) glaucoma $(n=1)$ (Table 1$)$. Preoperative ocular parameters reflected a substantial disease burden. Mean medicated IOP was $25.3 \pm 6.0 \mathrm{mmHg}$ on a mean of $2.98 \pm 0.88$ medications, with $75 \%(33 / 44)$ of eyes on $3-5$ glaucoma medications and no eyes medicationfree. Average visual field mean deviation (VF MD) was $-6.4 \mathrm{~dB}$, and mean $\mathrm{C}: \mathrm{D}$ ratio was 0.8 , with $73 \%$ of eyes $(32 / 44)$ having a C:D ratio $\geq$ 0.8 . Half of the eyes $(22 / 44)$ had undergone a total of 35 glaucoma surgeries prior to iStent inject implantation.

\section{Intraocular Pressure and Medication Use}

At 36 months postoperatively, mean IOP reduced by $42 \%$ to $14.6 \pm 2.0 \mathrm{mmHg}$ $(p<0.0001)$ (Fig. 3$)$, with $87.9 \%$ of eyes $(29 / 33)$ decreasing IOP by $\geq 20 \%$ versus preoperatively. Nearly all eyes $(97.0 \%$ or $32 / 33)$ achieved month 36 IOP $\leq 18 \mathrm{mmHg}$ versus $9.1 \%(4 / 44)$ preoperatively $(p<0.0001)$ (Fig. 4$)$, and $70.0 \%$ of eyes (23/33) achieved month 36 IOP $\leq 15 \mathrm{mmHg}$ versus $2.3 \%(1 / 44)$ preoperatively $(p<0.0001)$. Mean number of medications decreased by $82 \%$ to $0.55 \pm 0.79$ $(p<0.0001)$ (Fig. 5). The per-patient medication impact at 36 months consisted of $61 \%$ of eyes (20/33) becoming medication-free (versus 0\% preoperatively) and only $3.0 \%(1 / 33)$ on 3 medications versus $75 \%(33 / 44)$ on $3-5$ medications preoperatively (Fig. 6). All eyes maintained or decreased their 36-month medication burden versus preoperatively. 
Table 1 Demographics and preoperative characteristics ( $n=44$ eyes of 31 patients)

\begin{tabular}{ll}
\hline & $\begin{array}{l}\text { iStent } \text { inject (44 } \\
\text { eyes) }\end{array}$ \\
\hline Gender M/F & $23 / 21$ \\
Age (years) & $71.3 \pm 10.5$ \\
Mean \pm SD (range) & $(40-88)$ \\
Race & $100 \%$ Caucasian \\
C:D ratio & $0.8 \pm 0.1$ \\
Mean \pm SD (range) & $(0.5-1.0)$ \\
VF MD (dB) & $-6.4 \pm 6.9 \mathrm{~dB}$ \\
Mean \pm SD (range) & $(-24.4$ to -0.3$)$ \\
Type of glaucoma $n(\%)$ & \\
POAG & $38(86.4 \%)$ \\
PXG & $4(9.1 \%)$ \\
Appositional narrow angle & $1(2.3 \%)$ \\
Secondary (neovascular) & $1(2.3 \%)$ \\
Mean medicated IOP (mmHg); & $25.3 \pm 6.0 \mathrm{mmHg}$ \\
mean \pm SD & \\
Medicated IOP level (mmHg) $n(\%)$ & \\
$\leq 15$ mmHg & $1(2.3 \%)$ \\
$\leq 18$ mmHg & $4(9.1 \%)$ \\
Mean \# medications; mean $\pm S D$ & $2.98 \pm 0.88$ \\
Eyes with prior glaucoma surgery & $22(50.0 \%)$ \\
$n$ Prior glaucoma surgeries, $n=35$ surgeries \\
Trabeculectomy & 13 \\
CPC & 14 \\
ALT/SLT & 3 \\
Laser iridotomy & \\
XEN implantation & \\
\hline & \\
\hline &
\end{tabular}

Table 1 continued

\begin{tabular}{l}
\hline \multicolumn{1}{c}{$\begin{array}{c}\text { iStent } \text { inject (44 } \\
\text { eyes) }\end{array}$} \\
\hline $\begin{array}{l}\text { Eyes on 3-5 preoperative } \\
\text { medications, } n(\%)\end{array}$ \\
\hline$S D$ standard deviation, $C: D$ cup to disc, $V F M D$ visual \\
field mean deviation, $C P C$ cyclophotocoagulation, $A L T$ \\
argon laser trabeculoplasty, SLT selective laser \\
trabeculoplasty \\
a Eyes had open-angle configuration in the area of iStent \\
inject implantation \\
b Eyes could have more than one surgery (there were 35 \\
surgeries in 22 eyes)
\end{tabular}

\section{Safety}

Successful implantation of two second-generation trabecular micro-bypass stents was completed in all eyes in this study. One eye had planned XEN explantation at the time of stent surgery, while all other eyes underwent stent implantation as a sole procedure. One intraoperative complication was reported, consisting of mild hyphema in the anterior chamber, which necessitated no intervention and resolved without sequelae by week 1 .

Postoperatively, all but four eyes had CDVA of 20/50 or better throughout follow-up (26/26 eyes at 12 months, $21 / 21$ eyes at 24 months, and $30 / 30$ eyes at 36 months postoperatively). The four eyes with CDVA worse than 20/50 had preexisting poor CDVA (ranging from 20/63 to 20/20,000 Snellen equivalent preoperatively). Ocular adverse events through 36 months postoperatively included two eyes of the same patient that had cataract progression starting at month 3 (CDVA remained at 20/40 or better in both eyes through 36 months) and one eye with uveitis at 24 months, which resolved without sequelae with 3 weeks of topical anti-inflammatory medication. Two eyes underwent additional glaucoma procedures at 1 month postoperatively (XEN implant and cyclophotocoagulation, respectively) because of the need for additional IOP reduction beyond what was 


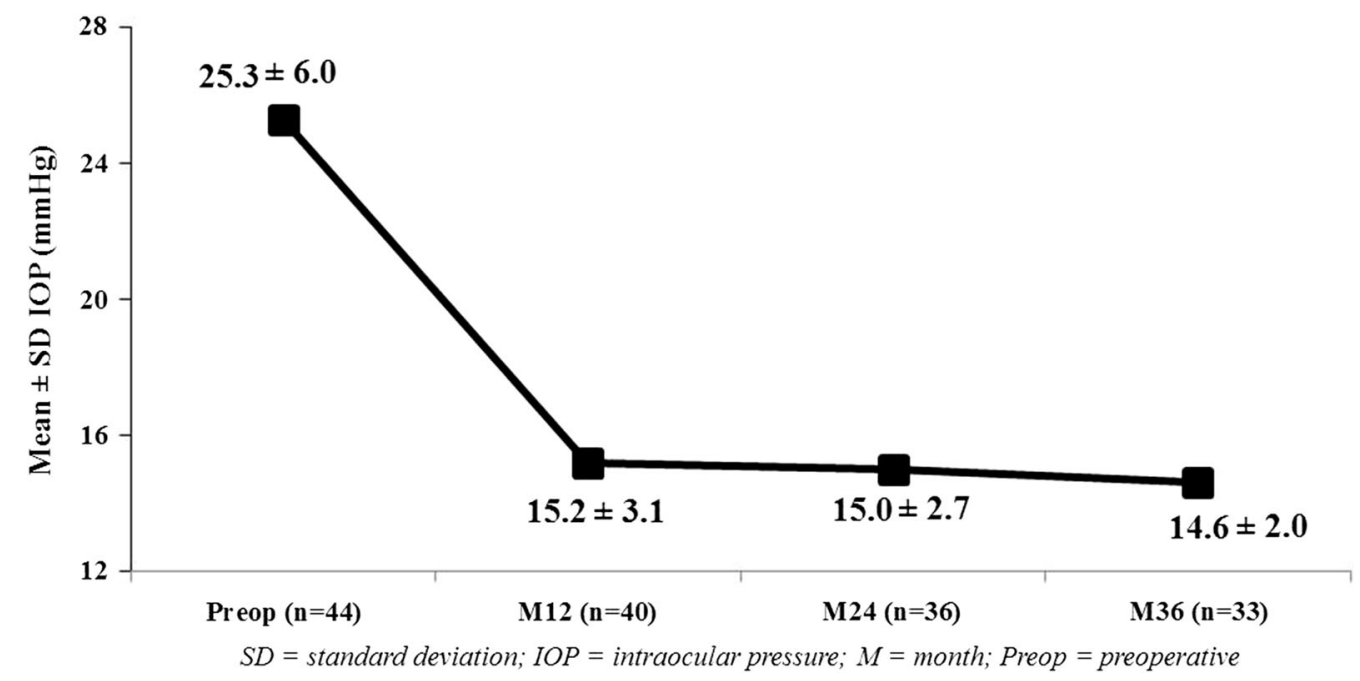

Fig. 3 Reduction in mean intraocular pressure through 36 months $(p<0.0001)$; eyes with available data at each visit. IOP intraocular pressure, $S D$ standard deviation, $M$ month, Preop preoperative

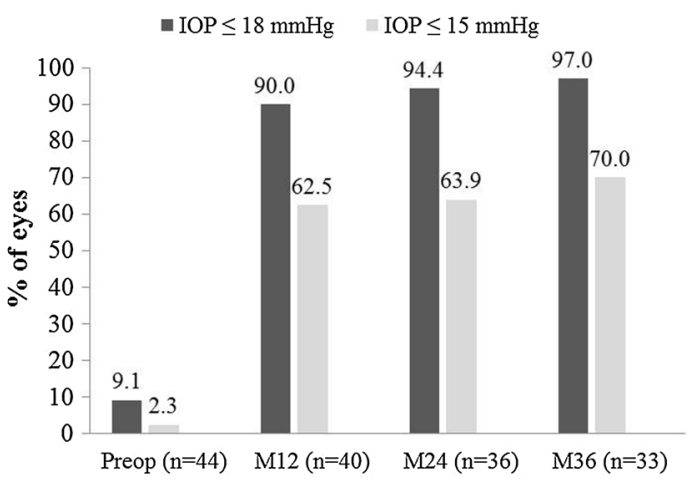

$I O P=$ intraocular pressure; $M=$ month; Preop = preoperative

*Highly significant $(p<0.0001)$ increase in proportion of eyes with $1 O P \leq 18 \mathrm{mmHg}$ and $I O P \leq 15 \mathrm{mmHg}$ at $M 36 \mathrm{vs}$. preoperative.

Fig. 4 Proportional analysis of eyes with IOP $\leq 18 \mathrm{mmHg}$ and $\leq 15 \mathrm{mmHg}$ through 36 months*; eyes with available data at each visit. IOP intraocular pressure, $M$ month, Preop preoperative. ${ }^{*}$ Highly significant $(p<0.0001)$ increase in proportion of eyes with IOP $\leq 18 \mathrm{mmHg}$ and $\mathrm{IOP} \leq 15 \mathrm{mmHg}$ at $\mathrm{M} 36$ vs. preoperatively

achieved with iStent inject implantation (IOP had reduced to $14 \mathrm{mmHg}$ ad $22 \mathrm{mmHg}$ in the two eyes, respectively). No other secondary glaucoma surgeries or ocular adverse events occurred for the remainder of follow-up. In particular, there were no reports of hypotony, endophthalmitis, corneal complications,

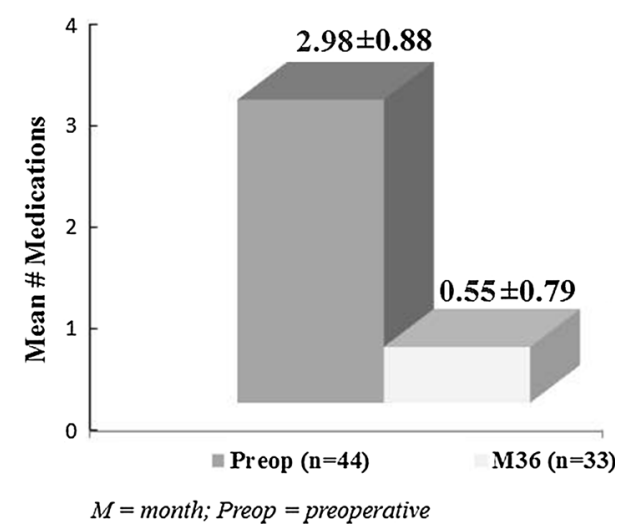

Fig. 5 Reduction in medication burden at 36 months vs. preoperative $(p<0.0001)$; eyes with available data at each visit. $M$ month, Preop preoperative

myopic shift, stent obstruction, peripheral anterior synechiae (PAS), or choroidal detachment.

\section{DISCUSSION}

Three-year data from this prospective real-world cohort demonstrated safe, sustained, clinically and statistically significant reductions in IOP and medications in eyes with glaucoma after stand-alone iStent inject implantation. The preoperative disease burden in this patient cohort 


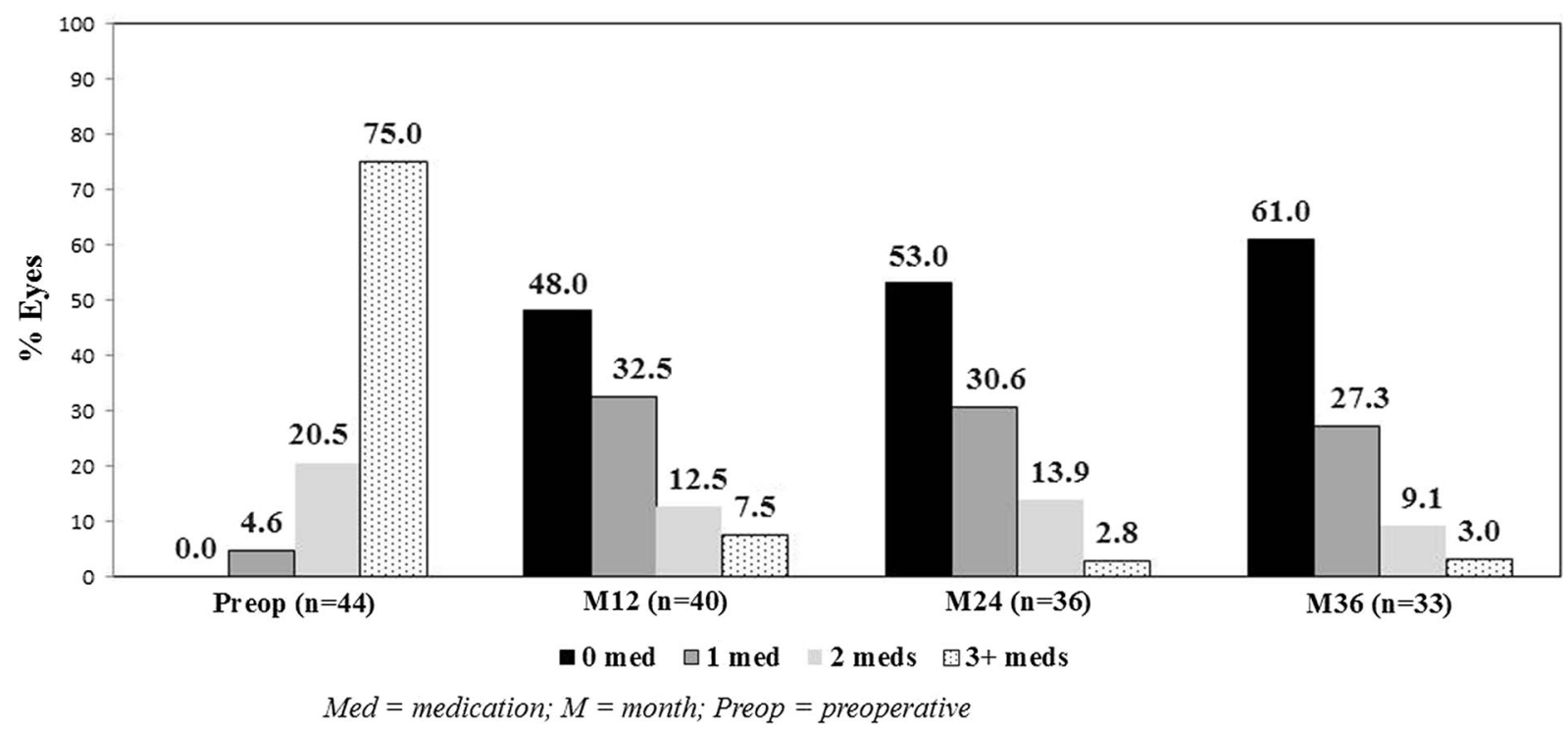

Fig. 6 Proportional analysis of medication use through 36 months; eyes with available data at each visit. $M$ month, Preop preoperative, Med medication

was sizable, with three-fourths of eyes on three or more medications, half of eyes with a history of prior glaucoma surgery, and moderate glaucomatous damage per VF MD and C:D ratio. Despite medications and/or surgery, however, mean preoperative IOP was $>25 \mathrm{mmHg}$, indicating the inability of such treatments to control the disease. It is noteworthy that iStent inject had a positive effect in these formerly treatment-resistant eyes. This treatment effect is consistent with prior studies showing benefit of this technology in various stages of glaucoma, from mild to advanced [40-51]. It also suggests that iStent inject may have a viable role in settings different from those typically considered for MIGS procedures: specifically, it may be considered not just in mild-to-moderate glaucoma as a primary surgery, but also in refractory eyes that have progressed despite previously undergoing more invasive surgery.

This study assessed stand-alone stent implantation, thereby allowing the device's treatment effects to be separated from those of phacoemulsification, which is known to reduce IOP to a modest degree in eyes with glaucoma [52-54]. Due to the stents alone, mean 36-month IOP showed a $42 \%$ decrease, a reduction consistent with those in previous studies of both combined and stand-alone
iStent inject implantation [40-51]. The observation of comparable 36-month outcomes either with or without cataract surgery is not entirely surprising, since the IOP-reducing effect of cataract surgery is known to be temporary [52-54] and thus would be unlikely to persist through the month 36 visit.

Compared with the previously published outcomes of the surgeon's stent-cataract experience, in which IOP was reduced by $37 \%$ and medications were reduced by $68 \%$ [46], the IOP and medication reductions in this stand-alone cohort were slightly higher (42\% and $82 \%$ reductions, respectively). Possible reasons for this difference include the higher preoperative IOP in the stand-alone cohort, as this is known to produce greater postoperative IOP reductions $[27,29]$. The preoperative medication burden also was higher in the stand-alone cohort, which may predispose to greater reductions in medication number if the end goal is the same for a given patient ( 0 or 1 medication, for example). The difference also may reflect the surgeon's learning curve, since the surgeon's first cases were mostly combination surgeries (iStent inject + phaco), whereas most of his stand-alone cases were completed later in his experience with the device. 
A particularly meaningful end point is the significant increase in eyes achieving IOP $\leq 18 \mathrm{mmHg}$ at 36 months $(97.0 \%$ versus $9.1 \%$ preoperatively), as reaching this IOP threshold is known to be associated with a lessened visual field decline over the long term in patients with glaucoma [55]. Coincident with this IOP decrease, mean medication burden was reduced by nearly 2.5 medications ( $82 \%$ reduction), and all eyes had maintained or reduced antihypertensive medications versus their preoperative regimen. While no eyes were medication-free preoperatively, a majority (61\%) were off all drops by 36 months postoperatively. While three-fourths of eyes were on 3-5 medications preoperatively, only one eye $(3.0 \%)$ was on 3 medications at 36 months postoperatively.

The benefits of medication reduction are myriad. It promotes treatment adherence, which has been shown to decrease dramatically when more than one eye drop is prescribed [5]. The ability to be off topical medications altogether is particularly advantageous, as considerable evidence has shown these medications to have toxic and pro-inflammatory effects on ocular surface cells $[4,10]$. Medication reduction lessens the financial burden of glaucoma treatment, for both patients and government bodies funding healthcare. This financial impact has been the area of several informative cost-effectiveness analyses in recent years [34-39]. The clinical outcomes in the present study are consistent with those reported in these financial evaluations. Thus, although this study did not analyze economic effects specifically, it is reasonable to expect that improved IOP control and a 2.43-medication reduction could result in similar benefits as those cited in these cost-effectiveness studies: for example, lower medication costs and provider expenditures, reduced societal burden from visual impairment, improved medication adherence, fewer IOP-related complications, and fewer quality-adjusted life years lost by patients because of poor vision [34-39]. Finally, topical medication usage has long been reported to cause patient-reported ocular surface discomfort and objective ocular surface damage $[4,10,11,56,57]$, raising the possibility that decreasing usage may positively impact patients' day-to-day life.

The long-term safety profile was favorable, including no significant intraoperative complications except for one case with slight hyphema that self-resolved without sequelae; postoperative adverse events that were few in number and mild in severity; and no serious ocular adverse events such as those seen with traditional filtering surgery or even some other MIGS devices [e.g., hypotony, endophthalmitis, corneal complications, myopic shift, stent obstruction, PAS, or choroidal detachment] [13-15, 58, 59]. Even though 3 years elapsed in this study, patients' visual acuity remained stable, with $100 \%$ of eligible eyes retaining CDVA of 20/50 or better through 36 months postoperatively. Two eyes underwent additional glaucoma procedures at 1 month postoperatively due to the need for further IOP lowering, but no other secondary glaucoma surgeries occurred throughout follow-up. This latter observation is notable considering the sizable preoperative disease burden of this cohort, as many of the eyes likely would have undergone filtration surgery if stent implantation were not performed.

Despite the study's strengths, several limitations may be discussed. The study design was non-randomized, unmasked, and consisted of single-surgeon, single-site data. As in nearly all real-world case series, no medication washouts were completed pre- or postoperatively, since these could place patients at undue risk that would not be indicated in standard clinical practice. For similar reasons, there was no control group to act as a counterpoint to the stentimplantation group; however, it is reasonable to treat patients' preoperative numeric data (such as IOP and number of medications) as viable comparators given the objective nature of these measures. This was a real-world, consecutive series, and thus no additional inclusion criteria were used outside of the surgeon's typical prerequisites for undergoing stent surgery. Future reports from this data set could include longer periods of follow-up; stratified outcomes by preoperative medications or prior glaucoma surgery; refractive data; and longitudinal 
analysis of the C:D ratio, VF, and OCT-measured retinal nerve fiber layer thickness.

\section{CONCLUSION}

In summary, this real-world prospective cohort of glaucomatous eyes with considerable preoperative disease burden achieved significant and sustained IOP and medication reductions through 36 months after stand-alone iStent inject implantation. Favorable long-term safety accompanied these outcomes, yielding a promising benefit-to-risk ratio that supports the viability of this treatment modality in eyes with mild to advanced glaucoma. Since outcomes were observed in a real-world clinical setting, the data can be informative for practicing surgeons and patients making treatment decisions.

\section{ACKNOWLEDGEMENTS}

We thank the participants in this study.

Funding. No funding was received for completing this study itself. Article processing charges and writing assistance were provided by Glaukos Corp. (San Clemente, CA, USA). All authors had full access to all of the data in this study and take complete responsibility for the integrity of the data and accuracy of the data analysis.

Authorship. All named authors meet the International Committee of Medical Journal Editors (ICMJE) criteria for authorship for this article, take responsibility for the integrity of the work as a whole, and have given their approval for this version to be published.

Meeting Presentation. Presented in part at the 2018 annual meetings of the American Society of Cataract and Refractive Surgery (ASCRS), the European Glaucoma Society (EGS), and the European Society of Cataract and Refractive Surgeons (ESCRS).

Disclosures. Christoffer Riffel has nothing to declare. Fritz Hengerer has received travel support, research grants, and lecture fees from Glaukos Corp. Gerd Auffarth has received travel support, research grants, and lecture fees from Glaukos Corp. Ina Conrad-Hengerer has received travel support, research grants, and lecture fees from Glaukos Corp.

Compliance with Ethics Guidelines. All procedures performed were in accordance with the ethical standards of the institutional and/or national research committee [the Institutional Review Board (IRB) of the University of Heidelberg] and with the 1964 Helsinki Declaration and its later amendments or comparable ethical standards. Informed consent was obtained from all individual participants included in the study.

Data Availability. The data sets generated during and/or analyzed during the current study are available from the corresponding author on reasonable request.

Open Access. This article is distributed under the terms of the Creative Commons Attribution-NonCommercial 4.0 International License (http://creativecommons.org/licenses/ by-nc/4.0/), which permits any noncommercial use, distribution, and reproduction in any medium, provided you give appropriate credit to the original author(s) and the source, provide a link to the Creative Commons license, and indicate if changes were made.

\section{REFERENCES}

1. Tham YC, Li X, Wong TY, Quigley HA, Aung T, Cheng CY. Global prevalence of glaucoma and projections of glaucoma burden through 2040: a systematic review and meta-analysis. Ophthalmology. 2014;121:2081-90.

2. Quigley HA. Glaucoma. Lancet. 2011;377:1367-77.

3. Lafuma A, Salmon JF, Robert J, Berdeaux G. Treatment persistence and cost-effectiveness of latanoprost/latanoprost-timolol, bimatoprost/ bimatoprost-timolol and travoprost/travoprost-timolol in glaucoma: an analysis based on the United Kingdom general practitioner research database. Clin Ophtlmol. 2011;5:361-7. 
4. Leung EW, Medeiros FA, Weinreb RN. Prevalence of ocular surface disease in glaucoma patients. J Glaucoma. 2008;17(5):350-5.

5. Robin AL, Novack GD, Covert DW, Crockett RS, Marcic TS. Adherence in glaucoma: objective measurements of once-daily and adjunctive medication use. Am J Ophthalmol. 2007;144(4):533-40.

6. Friedman DS, Quigley HA, Gelb L, et al. Using pharmacy claims data to study adherence to glaucoma medications: methodology and findings of the Glaucoma Adherence and Persistency Study (GAPS). Invest Ophthalmol Vis Sci. 2007;48(11):5052-7.

7. Nordstrom BL, Friedman DS, Mozaffari E, Quigley HA, Walker AM. Persistence and adherence with topical glaucoma therapy. Am J Ophthalmol. 2005;140(4):598-606.

8. Tsai JC. A comprehensive perspective on patient adherence to topical glaucoma therapy. Ophthalmology. 2009;116:S30-6.

9. Newman-Casey PA, Robin AL, Blachley T, et al. The Most Common Barriers to Glaucoma Medication Adherence: a Cross-Sectional Survey. Ophthalmology. 2015;122(7):1308-16.

10. Baudouin C, Liang H, Hamard P, et al. The ocular surface of glaucoma patients treated over the long term expresses inflammatory markers related to both T-helper 1 and T-helper 2 pathways. Ophthalmology. 2008;115(1):109-15.

11. Baudouin C, Labbé A, Liang H, Pauly A, BrignoleBaudouin F. Preservatives in eyedrops: the good, the bad and the ugly. Prog Retin Eye Res. 2010;29(4):312-34.

12. Glaucoma Laser Trial Research Group. The Glaucoma Laser Trial (GLT) and glaucoma laser trial follow-up study: 7. Results. Am J Ophthalmol. 1995;120:718-31.

13. Gedde SJ, Herndon LW, Brandt JD, Budenz DL, Feuer WJ, Schiffman JC. Tube versus trabeculectomy study group. postoperative complications in the tube versus trabeculectomy (TVT) study during five years of follow-up. Am J Ophthalmol. 2012;153:804-14.

14. Jampel HD, Musch DC, Gillespie BW, et al. Perioperative complications of trabeculectomy in the collaborative initial glaucoma treatment study (CIGTS). Am J Ophthalmol. 2005;140(1):16-22.

15. Rulli E, Biagioli E, Riva I, et al. Efficacy and safety of trabeculectomy vs nonpenetrating surgical procedures: a systematic review and meta-analysis. JAMA Ophthalmol. 2013;131(12):1573-82.
16. Pillunat LE, Erb C, Jünemann AG, Kimmich F. Micro-invasive glaucoma surgery (MIGS): a review of surgical procedures using stents. Clin Ophthalmol. 2017;11:1583-600.

17. Samuelson TW, Katz LJ, Wells JM, Duh YJ, Giamporcaro JE. Randomized evaluation of the trabecular micro-bypass stent with phacoemulsification in patients with glaucoma and cataract. Ophthalmology. 2011;118:459-67.

18. Craven ER, Katz LJ, Wells JM, Giamporcaro JE. Cataract surgery with trabecular micro-bypass stent implantation in patients with mild-to-moderate open-angle glaucoma and cataract: two-year followup. J Cataract Refract Surg. 2012;38:1339-45.

19. Fea AM, Consolandi G, Zola M, et al. Micro-Bypass implantation for primary open-angle glaucoma combined with phacoemulsification: 4-year followup. J Ophthalmol 2015; Volume 2015, Article ID 795357, 4 pages.

20. Arriola-Villalobos P, Martinez-de-la-Casa J, DiazValle D, Fernández-Pérez C, García-Sánchez J, García-Feijoó J. Combined iStent trabecular micro-bypass stent implantation and phacoemulsification for coexistent open-angle glaucoma and cataract: a long-term study. Br J Ophthalmol. 2012;96:645-9.

21. Belovay GW, Naqi A, Chan BJ, Rateb M, Ahmed II. Using multiple trabecular micro-bypass stents in cataract patients to treat open-angle glaucoma. J Cataract Refract Surg. 2012;38(11):1911-7.

22. Ferguson TJ, Berdahl JP, Schweitzer JA, Sudhagoni RG. Clinical evaluation of a trabecular micro-bypass stent with phacoemulsification in patients with open-angle glaucoma and cataract. Clinical Ophthalmology. 2016;10:1767-73.

23. Brown RH, Gibson Z, Zhong L, Lynch MG. Intraocular pressure reduction after cataract surgery with implantation of a trabecular microbypass device. J Cataract Refract Surg. 2015;41(6):1318-9.

24. Donnenfeld ED, Solomon KD, Voskanyan L, et al. A prospective 3-year follow-up trial of implantation of two trabecular microbypass stents in open-angle glaucoma. Clin Ophthalmol. 2015;9:2057-65.

25. Katz LJ, Erb C, Guillamet AC, Fea AM, Voskanyan L, Giamporcaro JE, Hornbeak DM. Long-term titrated IOP control with one, two, or three trabecular micro-bypass stents in open-angle glaucoma subjects on topical hypotensive medication: 42-month outcomes. Clin Ophthalmol. 2018;12:255-62.

26. Chang DF, Donnenfeld ED, Katz LJ, et al. Efficacy of two trabecular micro-bypass stents combined with topical travoprost in open-angle glaucoma not controlled on two preoperative medications: 3-year 
follow-up. Clinical Ophthalmology. 2017;11:523-8.

27. Ferguson TJ, Ibach M, Schweitzer J, Karpuk K, Stephens JD, Sudhagoni R, Berdahl JP. Trabecular microbypass stent implantation in pseudophakic eyes with open-angle glaucoma: Long-term results. J Cataract Refract Surg. 2019. https://doi.org/10. 1016/j.jcrs.2018.11.005.

28. Ferguson TJ, Swan R, Ibach M, Schweitzer J, Sudhagoni R, Berdahl JP. Trabecular microbypass stent implantation with cataract extraction in pseudoexfoliation glaucoma. J Cataract Refract Surg. 2017;43(5):622-6.

29. Ferguson T, Swan R, Ibach M, Schweitzer J, Sudhagoni R, Berdahl JP. Evaluation of a trabecular microbypass stent with cataract extraction in severe primary open-angle glaucoma. J Glaucoma. 2018;27(1):71-6.

30. Neuhann TH. Trabecular micro-bypass stent implantation during small-incision cataract surgery for open-angle glaucoma or ocular hypertension: long-term results. J Cataract Refract Surg. 2015;41:2664-71.

31. Vold SD, Voskanyan L, Tetz M, et al. Newly diagnosed primary open-angle glaucoma randomized to 2 trabecular bypass stents or prostaglandin: outcomes through 36 months. Ophthalmol Ther. 2016;5(2):161-72.

32. Buffet J, Brasnu E, Baudouin C, Labbé A. Efficacy of two trabecular micro-bypass stents during phacoemulsification for mild to advanced primary open-angle glaucoma controlled with topical hypotensive medications. J Glaucoma. 2017;26(12):1149-54.

33. Gallardo MJ, Supnet RA, Giamporcaro JE, Hornbeak DM. Outcomes of combined trabecular micro-bypass and phacoemulsification in a predominantly Hispanic patient population. Clin Ophthalmol. 2016;10:1931-7.

34. Berdahl JP, Khatana AK, Katz LJ, Herndon L, Layton AJ, Yu TM, Bauer MJ, Cantor LB. Cost-comparison of two trabecular micro-bypass stents versus selective laser trabeculoplasty or medications only for intraocular pressure control for patients with openangle glaucoma. J Med Econ. 2017;20(7):760-6.

35. Iordanous Y, Kent JS, Hutnik CM, Malvankar-Mehta MS. Projected cost comparison of Trabectome, iStent, and endoscopic cyclophotocoagulation versus glaucoma medication in the Ontario Health Insurance Plan. J Glaucoma. 2014;23(2):e112-8.

36. Ngan K, Fraser E, Buller S, Buller A. A cost minimization analysis comparing iStent accompanying cataract surgery and selective laser trabeculoplasty versus topical glaucoma medications in a public healthcare setting in New Zealand. Graefes Arch Clin Exp Ophthalmol. 2018. https://doi.org/10. 1007/s00417-018-4104-8 (Epub ahead of print).

37. Ordóñez JE, Ordóñez A, Osorio UM. Cost-effectiveness analysis of iStent trabecular micro-bypass stent for patients with open-angle glaucoma in Colombia. Curr Med Res Opin. 2018;10:1-12.

38. Tan SZ, Au L. Manchester iStent study: 3-year results and cost analysis. Eye 2016;1-6.

39. Wang SY, Singh K, Stein JD, Chang RT. Ocular Antihypertensive Medication Use After iStent Implantation Concurrent With Cataract Surgery vs Cataract Surgery Alone in a Large US Health Care Claims Database. JAMA Ophthalmol. 2018 Sep 27. https://doi.org/10.1001/jamaophthalmol.2018. 4461. (Epub ahead of print).

40. Fea AM, Belda JI, Rekas M, Junemann A, Chang L, Pablo L, Voskanyan L, Katz LJ. Prospective unmasked randomized evaluation of the iStent inject $^{\circledR}$ versus two ocular hypotensive agents in patients with primary open-angle glaucoma. Clinical Ophthalmology. 2014;8:875-82.

41. Klamann MKJ, Gonnermann J, Pahlitzsch M, et al. iStent inject in phakic open angle glaucoma. Graefes Arch Clin Exp Ophthalmol. 2015;253:941-7.

42. Lindstrom R, Lewis R, Hornbeak H, Voskanyan L, Giamporcaro JE, Hovanesian J, Sarkisian S. Outcomes Following Implantation of Two SecondGeneration Trabecular Micro-Bypass Stents in Patients with Open-Angle Glaucoma on One Medication: 18-Month Follow-Up. Adv Ther. 2016;33:2082-90.

43. Voskanyan L, Garcia-Feijoo J, Belda J, Fea A, Jünemann A, Baudouin C; Synergy Study Group. Prospective, Unmasked Evaluation of the iStent Inject System for Open-Angle Glaucoma: Synergy Trial. Adv Ther 2014 Feb;31(2):189-201.

44. Berdahl J, Voskanyan L, Myers JS, et al. Implantation of two second-generation trabecular microbypass stents and topical travoprost in open-angle glaucoma not controlled on two preoperative medications: 18-month follow-up. Clin Exp Ophthalmol. 2017;45(8):797-802.

45. Arriola-Villalobos P, Martinez-de-la-Casa J, DiazValle, Morales-Fernandez L, Fernandez-Perez C, Garcia-Feijoo J. Glaukos iStent inject trabecular micro-bypass implantation associated with cataract surgery in patients with co-existing cataract and open-angle glaucoma or ocular hypertension: A long-term study. J Ophthalmol 2016; https://doi. org/10.1155/2016/1056573. 
46. Hengerer FH, Auffarth GU, Riffel C, Conrad-Hengerer I. Prospective, non-randomized, 36-month study of second-generation trabecular micro-bypass stents with phacoemulsification in various types of glaucoma. Ophthalmol Ther. 2018;7(2):405-15.

47. Clement CI, Howes F, Ioannidis AS, Shiu M, Manning D. One-year outcomes following implantation of second-generation trabecular micro-bypass stents in conjunction with cataract surgery for various types of glaucoma or ocular hypertension: multicenter, multi-surgeon study Clinical Ophthalmology 2019:13 491-499.

48. Harasymowycz P. Single surgeon evaluation of second-generation trabecular micro-bypass stents in patients with mild to severe glaucoma. Platform presentation at: Annual meeting of the American Society of Cataract and Refractive Surgeons, 13-17 April 2018; Washington, DC: 2018.

49. Samuelson TW, Sarkisian Jr. SR, Lubeck DM, Stiles MC, Duh Y-J, Romo EA, Giamporcaro JE, Hornbeak DM, Katz LJ, for the iStent inject Study Group, Prospective, Randomized, Controlled Pivotal Trial of iStent inject Trabecular Micro-Bypass in Primary Open-Angle Glaucoma and Cataract: Two-Year Results. Ophthalmology 2019 Mar 14. pii: S01616420(18)31990-0. https://doi.org/10.1016/j.ophtha. 2019.03.006 (Epub ahead of print).

50. Guedes RAP, Gravina DM, Lake JC, Guedes VMP, Chaoubah A. Intermediate Results of iStent or iStent inject Implantation Combined with Cataract Surgery in a Real-World Setting: a Longitudinal Retrospective Study. Ophthalmol Ther. 2019;8(1):87-100. https://doi.org/10.1007/s40123019-0166-x (Epub 2019 Feb 5).

51. Popovic M, Campos-Moller X, Saheb H, K Ahmed II. Efficacy and Adverse Event Profile of the iStent and iStent Inject Trabecular Micro-bypass for Open-angle Glaucoma: A Meta-analysis. J Curr Glaucoma Pract. 2018 May-Aug;12(2):67-84. https:// doi.org/10.5005/jp-journals-10008-1248 (Epub 2018 Aug 1).
52. Vizzeri G, Weinreb RN. Cataract surgery and glaucoma. Curr Opin Ophthalmol. 2010;21(1):20-4.

53. Friedman DS, Jampel HD, Lubomski LH, et al. Surgical strategies for coexisting glaucoma and cataract: an evidence-based update. Ophthalmology. 2002;109:1902-13.

54. Mansberger SL, Gordon MO, Jampel $\mathrm{H}$, et al. Reduction in intraocular pressure after cataract extraction: the Ocular Hypertension Treatment Study. Ophthalmology. 2012;119:1826-31.

55. AGIS Investigators. The Advanced Glaucoma Intervention Study (AGIS): 7. The relationship between control of intraocular pressure and visual field deterioration. Am J Ophthalmol 2000;130: 429-40.

56. Bengtsson B, Heijl A, Johannesson G, AnderssonGeimer S, Aspberg J, Lindén C. The Glaucoma Intensive Treatment Study (GITS), a randomized clinical trial: design, methodology and baseline data. Acta Ophthalmol. 2018;96(6):557-66.

57. Zhang X, Vadoothker S, Munir WM, Saeedi O. Ocular surface disease and glaucoma medications: a clinical approach. Eye Contact Lens. 2018. https://doi.org/ 10.1097/icl.0000000000000544 (Epub ahead of print).

58. Schlenker MB, Gulamhusein H, Conrad-Hengerer I, Somers A, Lenzhofer M, Stalmans I, Reitsamer H, Hengerer FH, Ahmed IIK. Efficacy, safety, and risk factors for failure of standalone ab interno gelatin microstent implantation versus standalone trabeculectomy. Ophthalmology. 2017;124(11):1579_ 88.

59. Samuelson TW, Chang DF, Marquis R, Flowers B, Lim KS, Ahmed IIK, Jampel HD, Aung T, Crandall AS, Singh K; HORIZON Investigators. A Schlemm canal microstent for intraocular pressure reduction in primary open-angle glaucoma and cataract: the HORIZON study. Ophthalmology. 2018. 